\title{
BMJ A nationwide prospective cohort study Open on return to gainful occupation after stroke in Denmark 1996-2006
}

To cite: Hannerz H, Holbæk Pedersen B, Poulsen OM, et al. A nationwide prospective cohort study on return to gainful occupation after stroke in Denmark 1996-2006. BMJ Open 2011;1:e000180. doi:10 1136/bmjopen-2011-000180

- Prepublication history for this paper is available online. To view these files please visit the journal online (http:// bmjopen.bmj.com).

Received 13 May 2011 Accepted 19 July 2011

This final article is available for use under the terms of the Creative Commons Attribution Non-Commercial 2.0 Licence; see http://bmjopen.bmj.com

${ }^{1}$ National Research Centre for the Working Environment, Copenhagen, Denmark ${ }^{2}$ Centre of Rehabilitation of Brain Injury, Copenhagen, Denmark

Correspondence to Dr Harald Hannerz; hha@nrcwe.dk

\section{ABSTRACT}

Background: Return to work is an important outcome factor for working-age patients poststroke. Previous epidemiological studies on this topic have been small (on average 125 patients per study). Their estimated effects are therefore associated with a tremendous statistical uncertainty. The present study estimates the effect of various predictors on the odds of returning to work after stroke in the total population of 20-57-year-old previously employed hospital treated patients with stroke in Denmark 1996-2006 ( $\mathrm{N}=19$ 985).

Methods and results: The patients were followed through national registers; $62 \%$ were gainfully occupied 2 years after stroke. The odds of returning to work were higher among people with intracerebral infarction, OR 1.0 (the reference group), than they were among people with subarachnoid haemorrhage, OR $0.79(95 \% \mathrm{Cl} 0.71$ to 0.88$)$, and intracerebral haemorrhage, OR 0.39 (0.35 to 0.43 ). The odds of returning to work were lower among workers in elementary occupations OR 1.0 (reference group) than they were among workers in occupations that require skills at a basic level, OR 1.50 (1.38 to 1.64), technicians and associate professionals, OR 2.33 (2.05 to 2.65) and professionals, OR 3.04 (2.70 to 3.43 ). Patients in municipalities with a brain-injury rehabilitation centre did not have a better prognosis than patients in other municipalities, OR 0.91 (0.78 to 1.06). Being a woman, OR 0.79 (0.74 to 0.84 ), self-employed, OR 0.87 (0.78 to 0.96 ), or $\geq 50$ years, OR 0.61 (0.57 to 0.65 ), was associated with an adverse prognosis.

Conclusion: Further research is needed to explain the gender inequality, which suggests either a potential to improve return-to-work rates among the females or a tendency among the males to return too early.

\section{INTRODUCTION}

Return to work (RTW) plays a crucial role in the rehabilitation process of working-age patients poststroke. It provides a social identity, and it is associated with increased selfesteem and life satisfaction. ${ }^{1-3}$ It is also

\section{ARTICLE SUMMARY}

Article focus

- The present study estimates ORs for return to gainful occupation ca. 2 years after stroke.

- It focuses on clinical, demographic and occupational factors which are potentially useful in predicting return to work.

- The study includes the total population of 20-57-year-old previously employed hospital treated patients with stroke in Denmark 1996-2006.

Key messages

- $62 \%$ of the studied patients were gainfully occupied during the second calendar year after the stroke.

- The odds of returning to work depend on age gender, occupation, self-employment and type of stroke.

- Women had a much lower chance of returning to work than men, and to our knowledge there are no known physiological factors which can justify this difference.

- Patients in municipalities with a brain-injury rehabilitation centre did not have a better prognosis than patients in other municipalities.

Strengths and limitations of this study

- The follow-up was carried out through registers and all people in the target population were included. Hence, the study is free from sampling bias, recall bias and non-response bias.

- The statistical model was completely defined, and a detailed study protocol was published before we looked at any relation between the concerned exposure and response variables in our data material. Since we adhered to the protocol, the study is free from hindsight bias.

n The study is further strengthened by its size.

- The major weakness of the study is that it does not contain any stroke-severity measures.

important, from a societal perspective, that as many as possible of the patients with stroke who are economically active at the time of the onset RTW after stroke; in the European Union, the annual cost of lost productivity 
owing to disability or death as a consequence of stroke was estimated at $€ 8.5$ billion in 2008 . $^{4}$

A recent review of articles dealing with RTW among patients with stroke identified 70 studies, which in total comprised 8810 patients working before the stroke. ${ }^{5}$ The review concluded that the analytical strategies that were used were inappropriate in all but three of the 70 studies. ${ }^{6-8}$ The rest of the studies were associated with either selection bias or non-interpretable results owing to highly variable follow-up periods or ill-defined criteria of work before stroke and at follow-up. ${ }^{5}$ There are also limitations associated with the three appropriately performed studies. All of them were small (the included number of patients ranged from 109 to 173), and two of them ${ }^{6} 8$ deal with data that are too old to afford results that can be used as proxies for present-time RTW probabilities. The most recent of the three studies ${ }^{7}$ reported that $55 \%$ of previously employed patients with stroke in New Zealand returned to paid employment within 6 months. That study is, however, associated with selection bias; it only included cognitively competent patients.

The present study estimates the effect of various predictors on the odds of returning to work after stroke. It covers the total population of 20-57-year-old previously employed hospital treated patients with stroke in Denmark during 1996-2006.

\section{MATERIAL AND METHODS}

The study utilises the Danish Occupational Hospitalisation Register, a database obtained through a record linkage between three national registers - the central person register, the hospital patient register and the employment classification module. Currently, the Occupational Hospitalisation Register includes every person who has been economically active and an inhabitant of Denmark sometime after 1980.

The national hospital patient register has existed since 1977 and contains data from all public hospitals in Denmark (more than 99\% of all admissions). From 1977 to 1994 , the register included only inpatients, but from 1995 it also covered outpatients and emergency ward visits. ${ }^{9}$ The diagnoses have been coded according to international classification of diseases version 10 (ICD-10) since 1994.

The central person register contains information on gender, addresses and dates of birth, death and migrations for every person who is or has been an inhabitant of Denmark sometime between 1968 and the present. A person's occupation and social status have been, since 1975 , registered annually in the employment classification module. ${ }^{9}$ A person is classified according to his/her main income during the year. The occupations have been, since 1994, coded in accordance with Statistics Denmark's Standard Classification of Occupations (DISCO-88), ${ }^{10}$ which is a national version of the International Standard Classification of Occupations (ISCO-88). Socio-economic status is coded in accor- dance with Statistics Denmark's official socio-economic classification. ${ }^{11}$ At the one- and two-digit level, the classification contains the following social groups:

1. Gainfully occupied people

1.1. Self-employed people

1.2. Assisting spouses

1.3. Employees

2. People on unemployment benefits

3. Not economically active

3.1. People in training/education

3.2. Pensioners

3.3. Other not economically active

\section{Inclusion criteria}

A person would be included in the study if he/she

1. on at least one occasion in the time period 1996-2006 was registered in the hospital patient registry with one of the following ICD-10 codes as a principal diagnosis:

- I60 subarachnoid haemorrhage

- I61 intracerebral haemorrhage

- I63 cerebral infarction

- I64 stroke, not specified as haemorrhage or infarction

2. belonged to the age interval $20-57$ years at the time of the hospital contact

3. was gainfully occupied the year preceding the hospital contact.

\section{Accuracy of the databases}

Age and gender are part of the personal identification number of the central person register, which is recorded almost without errors. The completeness and accuracy of the data were confirmed in the matching process where we had a $100 \%$ match of the files. A person is classified according to his/her main income during the year. The socio-economic categories given above are based on incomes, which are declared to the Danish tax authority. This information should be correct among people who declare their income correctly. The occupational code, which is provided by the employer, is less reliable and is missing for almost $10 \%$ of the employees. For circulatory disease, the ICD-10 code of the principal diagnosis given in the hospital patient register has been estimated to be accurate in $73.4 \%$ of the records. ${ }^{12}$

\section{Ethics approval}

The study has been notified to and registered by Datatilsynet (the Danish Data Protection Agency). According to Danish law, questionnaire- and register-based studies do not need approval by ethical and scientific committees, or informed consent.

\section{Statistical analysis}

The study consists of two parts: one is descriptive, while the other utilises statistical inference techniques to test hypotheses and estimate ORs for RTW.

In the descriptive part, we followed the patients with stroke for five calendar years after the stroke for their 
main social status in each of these years. This part of the study only included people who were less than 55 years at the time of the stroke. For any given patient, the calendar year of the stroke was defined as year 0 , the next calendar year was defined as year 1 , etc.

In the regression analysis, we looked at the odds of having a socio-economic code, which indicates gainful occupation in year 2 after stroke. The outcome variable was set to 1 if the person was self-employed, assisting a spouse or an employee in that particular year. It was set to 0 if the person was unemployed, not economically active or dead.

As explanatory variables, we used gender, age, diagnosis, calendar year, occupational class, self-employment and type of municipality.

Age at the time of the stroke was divided into the categories $20-49$ years and 50-57 years. In Denmark, it is possible to retire at the age of 60 , regardless of health condition. Therefore, we excluded people being older than 60 years at follow-up. The cut-point 50 years conforms to the Organisation for Economic Co-operation and Development's definition of older workers, ${ }^{13}$ who are known to have a more insecure labour market attachment than younger workers.

The variable 'Diagnosis' contains the four stroke categories given in the section 'inclusion criteria.'

The variable 'Self-employment' was set to 1 if the person was self-employed or assisting a spouse and 0 if he/she was an employee, the year preceding the stroke.

The variable 'Occupational class' was based on the first digit of the DISCO-88 classification the year preceding the stroke. It contains the following categories:

- legislators, senior officials and managers (DISCO-88 group 1);

- professionals (DISCO-88 group 2);

- technicians and associate professionals (DISCO-88 group 3);

- workers in occupations that require skills at a basic level (DISCO-88 group 4-8);

- workers in elementary occupations (DISCO-88 group 9);

- gainfully occupied people with an unknown occupation (missing DISCO-88 code).

The variable 'Type of municipality' was set to 1 if the person lived in a municipality which had a brain-injury rehabilitation centre at the time of the stroke. Otherwise it was set to 0 . The following municipalities had a braininjury rehabilitation centre throughout the study period: Copenhagen, Odense, Aarhus, Roskilde, Aalborg and Vejle.

There are at least two reasons for believing that the RTW probabilities depended on which calendar year the stroke occurred. First, the quality of stroke treatment and rehabilitation has a tendency to improve with time. ${ }^{14-16}$ Second, a series of political initiatives and legislative changes, aimed at improving RTW rates in Denmark, occurred during the study period. ${ }^{17}$ We incorporated the calendar year into the model as a class variable to deal with this possible time dependency.
It is also reasonable to believe that RTW probabilities depend on place of residence. In Denmark, municipalities play an important role in the RTW process. According to the law, it is the municipal officer and not the physician who has the formal right to decide whether or not a person qualifies for sickness benefit, disability pension or vocational rehabilitation. The law also stipulates that the municipality should perform regular follow-up evaluations and draw up detailed reintegration plans for each sick-listed citizen at risk of long-term sickness absence. ${ }^{17}$ RTW initiatives are often launched at the municipality level, and some municipalities might be more active than others. The unemployment situations may also differ between municipalities. In the present study, we used a multilevel analysis to deal with intramunicipality correlations-the municipalities were treated as the subjects while the individual observations within the municipalities were treated as correlated repeated measurements.

The logistic regression was performed using the GENMOD procedure in SAS V.9.1. Only main effects were considered. We assumed an exchangeable correlation structure, and we used empirical SE estimates. The reference groups were chosen more or less arbitrarily, before we looked at any results. An OR which is higher than 1 indicates that the odds of returning to work are higher than they are in the reference group.

\section{RESULTS}

In total, 19985 patients fulfilled the inclusion criteria. Because of emigration, we missed follow-up data on 82 of them. The remaining 19903 patients were included in the analysis, and $12375(62.1 \%)$ of these were gainfully occupied during the second calendar year after the stroke. At baseline, $49.9 \%$ of the included patients were younger than 50 years old, and $39.1 \%$ were women.

The variable 'municipality type' did not reach statistical significance, but all other examined factors proved to be significant predictors of RTW. Patients with intracerebral infarction had a significantly higher chance of returning to work than patients with subarachnoid haemorrhage, who in turn had a significantly better prognosis than those with intracerebral haemorrhage. Patients who were employed before the stroke had a better prognosis than those who were self-employed; younger patients had a better prognosis than the older ones, and men had a better prognosis than women.

There was a clear relationship between the educational requirements of the job and the chance of returning to work after stroke. Professionals had a significantly better prognosis than technicians and associate professionals, who in turn had a significantly better prognosis than workers in occupations that only require skills at a basic level. The latter group had a significantly better prognosis than workers employed in elementary occupations, where no education is required. 
ORs for gainful occupation during the second calendar year after stroke are given in table 1 . Table 2 gives the social group distribution of the postpatients with stroke by time elapsed since the onset of illness. The category 'gainfully occupied' includes self-employed people, assisting spouses and employees. From table 2, the percentages in these categories are 5.3, 0.2 and 58.8 respectively. The sum of these percentages is not $62.1 \%$ but $64.3 \%$. The reason for the discrepancy is that table 2 only includes people in the age bracket 20-54 years, while the main analysis includes people in the age bracket $20-57$ years.

\section{DISCUSSION}

In the present study, we have shown the extent to which the odds for RTW after stroke depend on age, gender, occupational status, self-employment and type of stroke, and because of the size of the study we have done so with an extraordinary precision. The study did not require the participants to fill in a questionnaire; it was based solely on national registers. Recall bias and non-response bias were thereby eliminated. We also eliminated sampling bias by including the entire target population. Since the diagnoses studied require hospital treatment, referral bias should be minimal.

Bias owing to emigration should also be negligible, since only $0.4 \%$ of the subjects emigrated during the ca. 2-year follow-up period. The study is, moreover, free from hindsight bias; the statistical model was completely specified, and a study protocol was published ${ }^{19}$ before we looked at any relation between the exposure and response variables in our data material.

We had calendar-day-specific information on deaths, migrations and hospitalisations, but since the occupa- tional data only were given per calendar year, we did not have exact dates for RTW. Moreover, only the main occupation and social group during a calendar year are recorded in the register. A person could, in other words, RTW in a given calendar year and still be counted as a non-returner if he or she, for example, was absent because of sickness or unemployed during the major part of that year. Another consequence of not having a calendar-day-specific occupational history was that we had to resort to logistic regression instead of time-toevent analysis, which is regarded as the choice method in modelling RTW. ${ }^{5}$ Since RTW is not a rare event, the OR cannot be used as a proxy for the rate ratio, which makes it difficult to compare the effects obtained in the present study with those obtained in studies that use a timeto-event analysis. That the participants had to return to a fairly stable gainful occupation before they were categorised as having returned to work can, however, also be regarded as a strength. Studies show that many people with a brain injury RTW too soon and subsequently find that they are unable to continue their employment. ${ }^{1}$ Table 2 suggests that this is the case also for some of the people in the present study.

In the present study, we showed that people whose stroke was due to intracerebral haemorrhage, on average, had a worse prognosis than those with an intracerebral infarction. This finding is in agreement with previous research, which indicates that people with a stroke caused by haemorrhage tend to have more severe impairments at admission compared with those whose stroke is caused by an infarction. ${ }^{20-22}$ Stroke types, as given by the ICD-10 codes, are, however, quite crude proxies for stroke severity; it is obvious that a person with a minuscule intracerebral haemorrhage

Table 1 ORs, with $95 \% \mathrm{Cl}$ for return to gainful occupation 2 years after stroke

\begin{tabular}{|c|c|c|c|c|}
\hline Parameter & Level & $\mathbf{N}$ & Returns & OR $(95 \% \mathrm{Cl})$ \\
\hline \multirow[t]{2}{*}{ Gender } & Men & 12114 & 7698 & $1.00(-)$ \\
\hline & Women & 7789 & 4677 & $0.79(0.74$ to 0.84$)$ \\
\hline \multirow[t]{2}{*}{ Age } & $<50$ years & 9930 & 6608 & $1.00(-)$ \\
\hline & $50-57$ years & 9973 & 5767 & 0.61 (0.57 to 0.65$)$ \\
\hline \multirow[t]{4}{*}{ Diagnosis } & Subarachnoid haemorrhage & 3449 & 2070 & $0.79(0.71$ to 0.88$)$ \\
\hline & Intracerebral haemorrhage & 2272 & 976 & $0.39(0.35$ to 0.43$)$ \\
\hline & Cerebral infarction & 5952 & 3820 & $1.00(-)$ \\
\hline & $\begin{array}{l}\text { Stroke, not specified as haemorrhage } \\
\text { or infarction }\end{array}$ & 8230 & 5509 & $1.14(1.06$ to 1.23$)$ \\
\hline \multirow[t]{2}{*}{ Self-employment } & No & 17916 & 11180 & $1.00(-)$ \\
\hline & Yes & 1987 & 1195 & 0.87 (0.78 to 0.96$)$ \\
\hline \multirow[t]{6}{*}{ Occupational class } & Legislators, senior officials and managers & 1231 & 822 & 2.31 (2.00 to 2.68$)$ \\
\hline & Professionals & 2190 & 1624 & 3.04 (2.70 to 3.43$)$ \\
\hline & Technicians and associate professionals & 2980 & 2084 & 2.33 (2.05 to 2.65$)$ \\
\hline & $\begin{array}{l}\text { Workers in occupations that require skills } \\
\text { at a basic level }\end{array}$ & 9129 & 5478 & $1.50(1.38$ to 1.64$)$ \\
\hline & Workers in elementary occupations & 2475 & 1254 & $1.00(-)$ \\
\hline & Gainfully occupied people not otherwise specified & 1898 & 1113 & $1.28(1.15$ to 1.43$)$ \\
\hline \multirow[t]{2}{*}{ Municipality type } & Municipality without brain-injury centre & 15861 & 9908 & $1.00(-)$ \\
\hline & Municipality with brain-injury centre & 4042 & 2467 & $0.91(0.78$ to 1.06$)$ \\
\hline
\end{tabular}


Table 2 Social-group distribution (\%) by time elapsed since the onset of illness, among patients with stroke in Denmark 1996-2006 who were 20-54 years of age and gainfully occupied at the time of the stroke

\begin{tabular}{|c|c|c|c|c|c|}
\hline \multirow[b]{2}{*}{ Social status } & \multicolumn{5}{|c|}{ Year after stroke } \\
\hline & 1 & 2 & 3 & 4 & 5 \\
\hline Self-employed people & 6.1 & 5.3 & 4.9 & 4.7 & 4.7 \\
\hline Assisting spouses & 0.3 & 0.2 & 0.2 & 0.2 & 0.2 \\
\hline Employees & 64.9 & 58.8 & 55.7 & 53.6 & 51.4 \\
\hline People on unemployment benefits & 1.9 & 2.3 & 2.1 & 2.3 & 2.3 \\
\hline People in training/education & 0.4 & 0.4 & 0.4 & 0.2 & 0.2 \\
\hline Disability pensioners & 8.9 & 16.2 & 19.7 & 21.6 & 23.2 \\
\hline Other not economically active & 7.9 & 6.4 & 5.5 & 4.7 & 4.2 \\
\hline Deceased & 9.5 & 10.4 & 11.6 & 12.7 & 14.0 \\
\hline Total & 100.0 & 100.0 & 100.0 & 100.0 & 100.0 \\
\hline
\end{tabular}

might have a far better prognosis than someone with a massive cerebral infarction. In a prospective study on 1000 unselected patients with stroke in Denmark, ${ }^{20}$ stroke severity was the all-important prognostic factor; stroke type had no influence on neurological outcome when stroke severity was controlled for. Overall stroke severity is also the most consistent predictive factor for RTW. ${ }^{18}$ The major weakness of the present register study is that it lacks a direct severity measure, such as the American Heart Association Stroke Outcome Classification, ${ }^{23}$ the modified Rankin scale ${ }^{24}$ or the Barthel ADL Index. ${ }^{25}$ Our chances of correctly estimating whether or not an individual patient would RTW would have been considerably enhanced by such measures. The results of the present study are therefore more useful when viewed from a public-health perspective than they are from a clinical perspective. At the group level, the results can be used to estimate the proportion of patients that are expected to RTW. The study thereby provides a comparison material, which can be used by hospitals or municipalities, for example, to evaluate RTW programmes.

The prognosis among people with the ICD-10 code I64 (stroke, not specified as haemorrhage or infarction) is somewhat paradoxical. Stroke that is caused by either haemorrhage or infarction should not be associated with a better prognosis than both stroke specified as haemorrhage and stroke specified as infarction. The elevated odds for returning to work in this ill-defined patient group suggest that many of the people who were given the diagnosis I64 did not have a stroke in the first place.

The fact that men had a better chance of returning to work than women and the large difference between them is another paradoxical finding. Previous research does not indicate that female patients with stroke have a worse functional recovery than the male patients. ${ }^{26} 27$ It has, moreover, been shown that age-specific life expectancy after stroke is far better among women than among men. ${ }^{28}$ It is therefore reasonable to believe that at least a part of the gender difference observed in the present study is due to cultural, rather than physiological, factors. For example, in many cultures, a part of the male identity is to be responsible for providing for the household (being the breadwinner). In such cultures, a man who returns to work would regain not only his employment and stable income but also his male identity. ${ }^{29}$ Although Denmark is considered a modern country where men and women in many respects are treated as equals, attitudes and behaviour might still be influenced by the more traditional gender roles with regard to being a provider versus allowing oneself to be provided for. It is possible that these ancient gender roles influenced not only the patient but also the municipality official who is to decide whether or not a person qualifies for sickness benefit, disability pension or vocational rehabilitation. Since stress is believed to be an important risk factor for stroke, ${ }^{30}$ it is also possible that a part of the observed gender difference in RTW rates was due to a higher health and safety awareness and a lower propensity to take risks among women. ${ }^{31} 32$

Previous research on the relationship between occupational class and RTW after stroke was performed on patient samples that were too small to subdivide further than into white- versus blue-collar workers. The observed white- versus blue-collar ORs for RTW were 4.72 (USA, 1968-1973), 1.19 (USA, 1980s), 1.43 (Japan, 1986-1990) and 2.99 (Sweden, 1990s). ${ }^{2} 6833$ In the present study, we have shown that it is not only the physical requirements of the work (manual vs nonmanual) that matters but that inequalities also exist between occupational classes within the blue- and whitecollar categories. Although stroke is highly associated with cognitive impairments, ${ }^{34}$ we found that the probability of returning to work increased with the educational requirements of the job. This may sound counterintuitive at first. The investigated socio-occupational classes differ, however, not only in cognitive demands but also with respect to other psychosocial factors. There is, for example, a positive relationship between the educational requirements of the job and the possibilities to influence the volume as well as the content of ones work, ${ }^{35}$ and this is a factor that can play an important role in the RTW process. ${ }^{1}$ Educational requirements are also positively correlated with 'meaning of work.' 35

Patients in municipalities with a brain-injury rehabilitation centre did not have a better prognosis than 
patients in other municipalities. This null finding might be due to factors that have nothing to do with the centres. As only a minority of the patients are treated at a brain rehabilitation centre, our study cannot determine the effectiveness of the centres. It stresses, however, the importance of testing the workability of the centres in a randomised controlled trial before it is decided whether or not they should be recommended as an efficient RTW strategy.

It should finally be noted that the present study gives the situation in the Danish population and that the Danish system, where the eligibility for sickness benefit, vocational training and disability pension is determined by a municipal official, differs from that in many other countries where this is determined by a medical practitioner.

In conclusion, RTW strategies for patients poststroke ought to focus especially on the inequalities between the genders and socio-economic groups. Further research is needed to explain the gender inequality, which suggests either a potential to improve RTW rates among the females or a tendency among the males to return too early.

Acknowledgements S Vester Thorsen, K Carlsen, M Haulund Andersen, $T$ Lehmann Lund and J Bue Bjørner at the National Research Centre for the Working Environment (NRCWE) are thanked for valuable discussions. E Bengtsen and R Nilsson, NRCWE, are thanked for their assistance in the obtainment of references.

Correction notice The "To cite: ..." information and running footer in this article have been updated with the correct volume number (volume 1).

Funding The study was funded by The Danish National Labour Market Authority, grant number 2008-5231.

Competing interests FH is Director of the Centre for Rehabilitation of Brain Injury (CRBI) in Copenhagen, Denmark. CRBI is a self-owned fund that is financially supported by grants from the Danish municipalities and, to a lesser degree, by a collectively bargained framework agreement under the Danish Health Law that covers $20 \%$ of the funds' operation costs. Since the study could tell us whether or not patients with stroke in municipalities with brain-injury rehabilitation centres have a better prognosis than those in other municipalities, there was a potential conflict of interest. We believe, however, that any potential bias owing to competing interests was eliminated by the publication of our detailed study protocol, which implied a commitment to adhere to the methods chosen and to publish the results regardless of the outcome.

Contributors LLA, OMP and FH initiated the project and acquired the funding. LLA, OMP and HH designed the study. HH performed the statistical analysis. $\mathrm{HH}$ and BHP prepared the first draft of the manuscript. All authors contributed to a critical revision of the manuscript.

Provenance and peer review Not commissioned; externally peer reviewed.

Data sharing statement The study was based on national registers. Questions regarding the usage of these registers should be addressed to Statistics Denmark.

\section{REFERENCES}

1. Wolfenden B, Grace M. Returning to work after stroke: a review. Int J Rehabil Res 2009;32:93-7.

2. Vestling $\mathrm{M}$, Tufvesson $\mathrm{B}$, Iwarsson $\mathrm{S}$. Indicators for return to work after stroke and the importance of work for subjective well-being and life satisfaction. J Rehabil Med 2003;35:127-31.

3. Shames J, Treger I, Ring $\mathrm{H}$, et al. Return to work following traumatic brain injury: trends and challenges. Disabil Rehabil 2007;29:1387-95.

4. Di CA. Human and economic burden of stroke. Age Ageing 2009;38:4-5.
5. Daniel K, Wolfe CD, Busch MA, et al. What are the social consequences of stroke for working-aged adults? A systematic review. Stroke 2009;40:e431-40.

6. Saeki S, Ogata H, Okubo T, et al. Return to work after stroke. A follow-up study. Stroke 1995;26:399-401.

7. Glozier N, Hackett ML, Parag V, et al. The influence of psychiatric morbidity on return to paid work after stroke in younger adults: the Auckland Regional Community Stroke (ARCOS) Study, 2002 to 2003. Stroke 2008;39:1526-32.

8. Wozniak MA, Kittner SJ, Price TR, et al. Stroke location is not associated with return to work after first ischemic stroke. Stroke 1999;30:2568-73.

9. Soll-Johanning $\mathrm{H}$, Hannerz $\mathrm{H}$, Tuchsen $\mathrm{F}$. Referral bias in hospital register studies of geographical and industrial differences in health. Dan Med Bull 2004;51:207-10.

10. Statistics Denmark. DISCO-88 Statistics Denmark's Standard Classification of Occupations. Copenhagen: Statistics Denmark, 1996.

11. Statistics Denmark. SOCIO Danmarks Statistiks Socioøkonomiske Klassifikation. 1st edn. Copenhagen: Statistics Denmark, 1997.

12. Nickelsen TN. Data validity and coverage in the Danish National Health Registry. A literature review. Ugeskr Laeger 2001;164:33-7.

13. Keese M. Live Longer, Work Longer. Aging and Employment Policies. Paris: OECD Publications, 2006.

14. Immonen-Raiha $\mathrm{P}$, Mahonen $\mathrm{M}$, Tuomilehto $\mathrm{J}$, et al. Trends in casefatality of stroke in Finland during 1983 to 1992. Stroke 1997;28:2493-9.

15. Pajunen $\mathrm{P}$, Paakkonen $\mathrm{R}$, Hamalainen $\mathrm{H}$, et al. Trends in fatal and nonfatal strokes among persons aged 35 to $>$ or $=85$ years during 1991-2002 in Finland. Stroke 2005;36:244-8.

16. Roberts SE, Goldacre MJ. Case fatality rates after admission to hospital with stroke: linked database study. BMJ 2003;326:193-4.

17. Johansen $\mathrm{K}$, Andersen JS, Mikkelsen S, et al. Controlling sickness absence: a study of changes in the Danish sickness absence legislation since 1973. Health Policy 2008;86:109-18.

18. Treger I, Shames J, Giaquinto S, et al. Return to work in stroke patients. Disabil Rehabil 2007;29:1397-403.

19. Hannerz H, Pedersen BH, Poulsen OM, et al. Study protocol to a nationwide prospective cohort study on return to gainful occupation after stroke in Denmark 1. BMC Public Health 2010;10:623.

20. Jorgensen $\mathrm{HS}$, Nakayama $\mathrm{H}$, Raaschou $\mathrm{HO}$, et al. Intracerebral hemorrhage versus infarction: stroke severity, risk factors, and prognosis. Ann Neurol 1995;38:45-50.

21. Kelly PJ, Furie KL, Shafqat $\mathrm{S}$, et al. Functional recovery following rehabilitation after hemorrhagic and ischemic stroke. Arch Phys Med Rehabil 2003;84:968-72.

22. Katrak PH, Black D, Peeva V. Do stroke patients with intracerebral hemorrhage have a better functional outcome than patients with cerebral infarction? PM $R$ 2009;1:427-33

23. Kelly-Hayes M, Robertson JT, Broderick JP, et al. The American Heart Association Stroke Outcome Classification. Stroke 1998;29:1274-80.

24. van Swieten JC, Koudstaal PJ, Visser MC, et al. Interobserver agreement for the assessment of handicap in stroke patients. Stroke 1988; 19:604-7.

25. Collin C, Wade DT, Davies S, et al. The Barthel ADL Index: a reliability study. Int Disabil Stud 1988;10:61-3.

26. Wade DT, Hewer RL, Wood VA. Stroke: influence of patient's sex and side of weakness on outcome. Arch Phys Med Rehabil 1984;65:513-16.

27. Bonita R, Beaglehole R. Recovery of motor function after stroke. Stroke 1988;19:1497-500.

28. Hannerz H, Nielsen ML. Life expectancies among survivors of acute cerebrovascular disease. Stroke 2001;32:1739-44.

29. Bouta T, Frekrs G, Bannon I. Gender, Conflict and Development. Washington, DC: World Bank Publications, 2004.

30. Truelsen $\mathrm{T}$, Nielsen N, Boysen $\mathrm{G}$, et al. Self-reported stress and risk of stroke: the Copenhagen City Heart Study. Stroke 2003;34:856-62.

31. Powell M, Ansic D. Gender difference in risk behaviour in financial decision-making: an experimental analysis. J Econ Psychol 1997;18:605-28.

32. Wilson M, Daly M, Gordon S, et al. Sex differences in valuations of the environment? Popul Environ 1996;18:143-59.

33. Howard G, Till JS, Toole JF, et al. Factors influencing return to work following cerebral infarction. JAMA 1985;253:226-32.

34. Hoffmann M. Higher cortical function deficits after stroke: an analysis of 1,000 patients from a dedicated cognitive stroke registry. Neurorehabil Neural Repair 2001;15:113-27.

35. Moncada S, Pejtersen JH, Navarro A, et al. Psychosocial work environment and its association with socioeconomic status. A comparison of Spain and Denmark. Scand J Public Health 2010;38:137-48. 